\title{
Caspase-2 mediates triglyceride (TG)-induced macrophage cell death
}

\author{
Jaewon Lim ${ }^{1,2, \#}$, Hyun-Kyung Kim ${ }^{1,3, \#, ~ S u n g ~ H o o n ~ K i m ~}{ }^{1}$, Ki-Jong Rhee ${ }^{1} \mathcal{E}$ Yoon Suk Kim ${ }^{1, *}$ \\ ${ }^{1}$ Department of Biomedical Laboratory Science, College of Health Sciences, Yonsei University, Wonju 26493, ${ }^{2}$ Department of Clinical \\ Laboratory Science, College of Medical Sciences, Daegu Haany University, Gyeongsan 38610, ${ }^{3}$ Department of Biomedical Laboratory \\ Science, College of Natural Science, Gimcheon University, Gimcheon 39528, Korea
}

Triglyceride (TG) accumulation causes macrophage cell death, which affects the development of atherosclerosis. Here, we examined whether caspase-2 is implicated in TG-induced macrophage cell death. We found that caspase-2 activity is increased in TG-treated THP-1 macrophages, and that inhibition of caspase-2 activity drastically inhibits TG-induced cell death. We previously reported that TG-induced macrophage cell death is triggered by caspase-1, and thus investigated the relationship between caspase-2 and caspase-1 in TG-induced macrophage cell death. Inhibition of caspase-2 activity decreased caspase-1 activity in TG-treated macrophages. However, caspase-1 inhibition did not affect caspase-2 activity, suggesting that caspase-2 is upstream of caspase-1. Furthermore, we found that TG induces activation of caspase-3, $-7,-8$, and -9, as well as cleavage of PARP. Inhibition of caspase-2 and -1 decreased TG-induced caspase-3, -7, -8, and -9 activation and PARP cleavage. Taken together, these results suggest that TG-induced macrophage cell death is mediated via the caspase-2/caspase-1/apoptotic caspases/PARP pathways. [BMB Reports 2017; 50(10): 510-515]

\section{INTRODUCTION}

Triglycerides (TGs) are a type of lipid that can cause inflammation and lead to chronic vascular disease (1). The uptake of TGs by macrophages is an important contributing factor during the progression of inflammatory vascular diseases (2). TGs have been proposed to induce foam cell formation,

*Corresponding author. Tel: +82-33-760-2860; Fax: +82-33-7602195; E-mail: yoonsukkim@yonsei.ac.kr

${ }^{\text {"}}$ These authors contributed equally to this work.

https://doi.org/10.5483/BMBRep.2017.50.10.106

Received 22 June 2017, Revised 11 July 2017, Accepted 1 August 2017

Keywords: Apoptotic caspases, Caspase-1, Caspase-2, THP-1 macrophages, Triglyceride (TG) resulting in increased programmed cell death (PCD) of macrophages, and inflammatory reactions in vascular diseases $(3,4)$. The process of PCD, which includes apoptosis and pyroptosis, is generally characterized by distinct morphological characteristics and various biochemical mechanisms.

Apoptosis involves the activation of a group of cysteine proteases, known as apoptotic caspases, that mediate DNA cleavage. Apoptotic cells are targeted for phagocytosis and removal, resulting in an absence of inflammation and lack of cytokine secretion $(5,6)$. Pyroptosis is mechanistically distinct from apoptosis, causing inflammatory cell death commonly mediated by the activation of caspase- $1(6,7)$. Caspase- 1 induces the release of proinflammatory cytokines (IL-1 $\beta$ and IL-18) and rapid plasma-membrane rupture, leading to cell lysis. Several reports have shown that caspases mediating DNA damage during apoptosis also participate in DNA damage during pyroptosis $(8,9)$.

Classical apoptotic caspases are categorized as either initiator caspases (caspase-8 and caspase-9) or effector caspases (caspase-3 and caspase-7) $(10,11)$. Effector caspases are cleaved and activated by activated initiator caspases. Activated effector caspases also increase the cleavage of poly ADP ribose polymerase (PARP), a hallmark of apoptotic cell death (10-12). In addition, apoptosis occurs through two main apoptotic pathways: the extrinsic pathway, in which caspase-8 is mainly involved, and the intrinsic (mitochondrial) pathway, in which caspase-9 is mainly activated $(5,12)$.

Previous studies have shown that intracellular TG accumulation influences mitochondria in order to activate intrinsic pathway-associated apoptotic caspases in macrophages $(3,13)$. In addition, loading macrophages with TGs has been reported to induce caspase-1-dependent pyroptotic cell death (14). Although caspase-1 has been reported to play a role in TG-triggered macrophage cell death, neither its upstream molecules nor its downstream mediators have been identified in detail. Caspase- 1 is involved in the intrinsic and extrinsic apoptotic pathways in a variety of stimuli-induced cell death $(12,15,16)$. However, it remains unclear whether caspase- 1 is associated with the apoptotic pathway as well as the pyroptotic pathway in TG-induced macrophage cell death.

Caspase-2 is a highly conserved, but poorly understood 
member of the caspase family $(17,18)$. Caspase-2 has been implicated in PCD induced by multiple intrinsic and extrinsic stimuli, including DNA damage, reactive oxygen species (ROS), and cytoskeletal disruption (17). However, it is unknown whether caspase-2 is involved in TG-stimulated macrophage cell death. According to recent studies, caspase-2 is associated with activation of caspase-1, as well as interleukin-1 $\beta$ (IL-1 $\beta$ ) secretion (6), and the chemical inhibition of caspase- 2 decreases the activation of caspase- 1 and cell death in Salmonella-infected macrophages (9). Meanwhile, several studies have reported that activated caspase-2 mediates the activation of both caspase- 3 and -8 , as well as the secretion of tumor necrosis factor- $\alpha$ (TNF- $\alpha$ ) in macrophages $(6,19)$.

In this study, we investigated both the involvement of caspase-2 in TG-induced macrophage cell death, and the association of caspase- 2 with caspase- 1 and classical apoptotic caspases in TG-treated THP-1 macrophage cells. We report here that caspase- 2 mediates TG induced-macrophage cell death by activating caspase- 1 and classical apoptotic caspases.

\section{RESULTS}

\section{Caspase-2 is implicated in TG-induced THP-1 macrophage cell death}

Previous studies have reported that caspase- 2 is associated with a variety of stimuli-induced cell death $(5,6,9)$, but its involvement in TG-induced macrophage cell death remains unclear. To determine whether caspase-2 is involved in TG-stimulated macrophage cell death, we reconfirmed that TG induces decreased cell viability in THP-1 macrophages. As shown in Fig. 1A, treatment with TG for $24 \mathrm{~h}$ reduced cell viability in THP-1 macrophages. Next, we examined the activity of caspase-2 in TG-treated THP-1 macrophages. PMA-differentiated THP-1 cells were treated with TG for different times $(0,3,6,12,18,24 \mathrm{~h})$ and caspase-2 activity was analyzed. Caspase-2 activity was increased in THP-1 macrophages exposed to TG in a time-dependent manner (Fig. 1B). To elucidate whether the increased caspase- 2 activity was responsible for macrophage cell death, THP-1 macrophages were treated with TG in the presence or absence of z-VDVAD-FMK, a caspase-2 specific inhibitor, for $24 \mathrm{~h}$ and viable cells were enumerated. Treatment with z-VDVAD-FMK inhibited TG-induced macrophage cell death in an inhibitor dose-dependent manner (Fig. 1C). In addition, when caspase-2 was knocked down by siRNA in THP-1 macrophages, TG-induced macrophage cell death was decreased (Fig. 1D, E). These results suggest that caspase- 2 is involved in TGstimulated macrophage cell death.

\section{Caspase-2 induces activation of caspase-1 in TG-stimulated THP-1 macrophages cell death}

We recently showed that TG-induced macrophage cell death is mediated by caspase-1 (14). We confirmed the involvement of caspase-1 in TG-stimulated macrophage cell death by
A

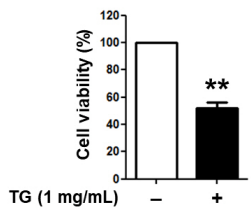

B

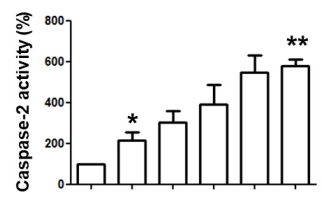

TG (1 mg/mL) $\begin{array}{lllllllll}0 & 3 & 6 & 12 & 18 & 24 & \text { (h) }\end{array}$

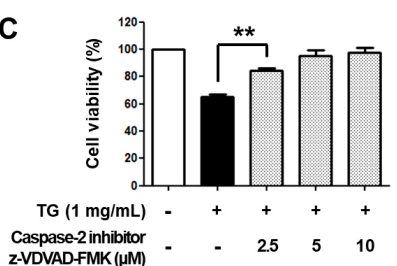

D

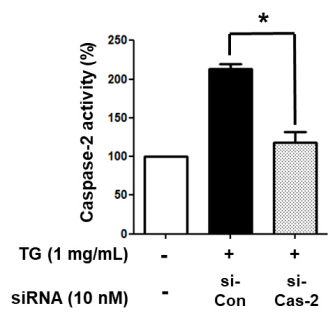

E

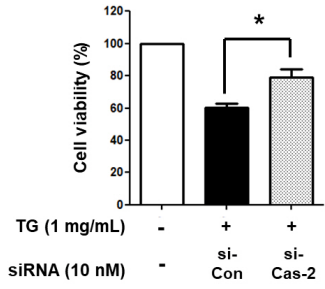

Fig. 1. Caspase-2 is involved in TG-induced THP-1 macrophage cell death. (A) THP-1 cells were differentiated with $200 \mathrm{nM}$ PMA for $48 \mathrm{~h}$ and incubated with or without TG for an additional 24 h. Viable cells were enumerated by trypan blue dye exclusion assay. The number of viable cells in THP- 1 macrophages without TG treatment was set as $100 \%$. (B) THP-1 macrophages were incubated with TG for the indicated times, after which caspase-2 activity was assessed. The absorbance of THP-1 macrophages without TG treatment was set as $100 \%$. (C) THP-1 macrophages were incubated with TG in the presence of the caspase-2 inhibitor z-VDVAD-FMK for $24 \mathrm{~h}$. Viable cells were enumerated. The number of viable cells in THP-1 macrophages without TG treatment was set as $100 \%$. THP-1 macrophages were transiently transfected with the control siRNA (si-Con) or caspase-2 siRNA (si-Cas-2) for $24 \mathrm{~h}$ and incubated with TG for an additional $24 \mathrm{~h}$. (D) Caspase-2 activity was assessed. The absorbance of THP-1 macrophage without siRNA transfection and TG treatment was set as $100 \%$. (E) Viable cells were enumerated. The number of viable cells in THP-1 macrophages without siRNA transfection and TG treatment was set as $100 \%$. Data are expressed as the mean \pm SEM of three independent experiments. The $\mathrm{P}$ values were determined with Student's $t$-test. ${ }^{*} P<0.05,{ }^{*} * \mathrm{P}<0.01$.

treating THP-1 macrophages with TG for $24 \mathrm{~h}$. As expected, the activity of caspase- 1 was increased in TG-treated THP-1 macrophages (Fig. 2A) and cleaved caspase-1 (i.e., activated caspase-1) was also increased in a time-dependent manner (Fig. 2B).

Caspase-2 is involved in the activation of caspase- 1 in bacteria-induced cell death $(6,9)$. Therefore, we examined the association of caspase- 2 with caspase-1 activation in TG-induced macrophage cell death. To this end, THP-1 macrophages were treated with TG in the presence of the caspase-2 inhibitor $\mathrm{z}$-VDVAD-FMK for $24 \mathrm{~h}$ and caspase- 1 activity was determined. Caspase-1 activity and cleaved caspase-1 levels were decreased by the inhibitor in a dose-dependent manner (Fig. 2C, D). In addition, when caspase- 2 was knocked down by siRNA in 
A

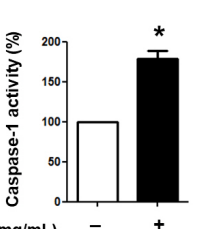

B

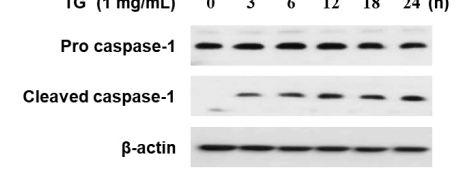

C

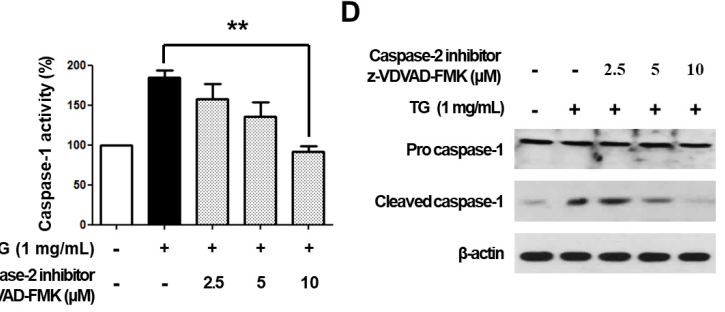

E

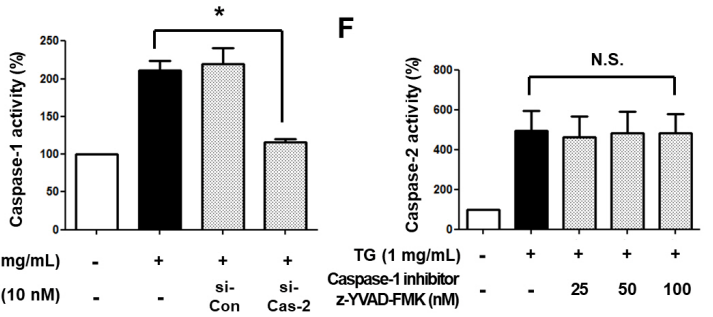

Fig. 2. Caspase-2 is an upstream molecule of caspase-1 in TGstimulated cell death of THP-1 macrophages. (A) THP-1 macrophages were treated with or without TG for $24 \mathrm{~h}$ and caspase- 1 activity was assessed. The absorbance of THP-1 macrophages without TG treatment was set as $100 \%$. (B) THP-1 macrophages were treated with TG for the indicated times. Cleavage of caspase-1 was detected by Western blotting. $\beta$-actin was used as an internal control. (C) THP-1 macrophages were treated with TG in the presence of the caspase- 2 specific inhibitor z-VDVAD-FMK for 24 $h$, after which caspase- 1 activity was assessed. The absorbance of THP-1 macrophages without TG treatment was set to $100 \%$. (D) Cleaved caspase-1 was detected by Western blotting. (E) THP-1 macrophages were transfected with control siRNA or caspase-2 siRNA for $24 \mathrm{~h}$ and incubated with or without TG for an additional $24 \mathrm{~h}$. Caspase- 1 activity was assessed. The absorbance of THP-1 macrophage without TG treatment was set as $100 \%$. (F) THP-1 macrophages were treated with TG in the presence of the caspase-1 specific inhibitor z-YVAD-FMK for $24 \mathrm{~h}$ and the caspase-2 activity was assessed. All data are expressed as the mean \pm SEM of three independent experiments. $P$ values were determined with Student's $t$-test. ${ }^{*} \mathrm{P}<0.05,{ }^{*} * \mathrm{P}<0.01$.

THP-1 macrophages, TG-induced increase of caspase-1 activity was inhibited (Fig. 2E). On the other hand, when THP-1 macrophages were treated with TG in the presence of the caspase-1 inhibitor z-YVAD-FMK, we observed no significant changes in caspase-2 activity in cells in response to TGs (Fig. 2F). Taken together, these results indicate that caspase- 2 is upstream of caspase- 1 , and is associated with its activation in TG-induced THP-1 macrophage cell death.

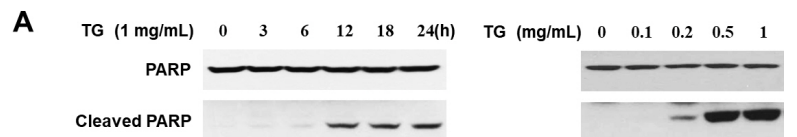

B

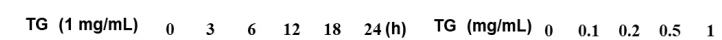

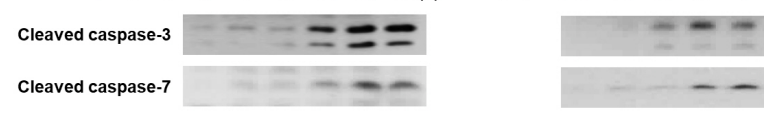

C

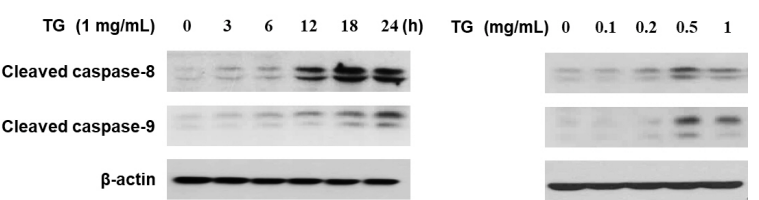

Fig. 3. TG treatment induces the cleavage of apoptotic caspases and PARP in THP-1 macrophages. THP-1 macrophages were treated with the indicated concentration of TG for the indicated times. (A) The cleaved form of PARP, (B) cleavage of caspase-3 and -7 , and $(C)$ cleavage of caspase- 8 and -9 was detected by Western blotting.

The cleavage of apoptotic caspases and PARP is induced in TG-stimulated macrophage cell death

It has been reported that TG accumulation induces dysfunction of mitochondria and activation of intrinsic pathway-associated caspases (13). In addition, PARP is cleaved and inactivated during caspase-dependent cell death (12). Thus, we investigated whether PARP and apoptotic caspases are modulated in response to TG in THP-1 macrophages. THP-1 macrophages were treated with concentrations of TG $(0,0.1,0.2,0.5$, and 1 $\mathrm{mg} / \mathrm{ml})$ for the indicated times $(0,3,6,12,18,24 \mathrm{~h})$. We first examined cleavage of PARP in TG-stimulated THP-1 macrophages. As shown in Fig. 3A, PARP cleavage was increased in a TG dose- and time-dependent manner. Next, we investigated activation of caspase-3 and caspase-7, which are known to cleave PARP (12). TG treatment induced the cleavage of both caspase- 3 and caspase- 7 in a TG dose- and time-dependent manner (Fig. 3B). Caspase-8 and caspase-9 are upstream molecules of caspase- 3 and -7 , therefore we also examined the activation of caspase- 8 and -9 , and found that both caspase- 8 and -9 are cleaved in TG-treated THP-1 macrophages (Fig. 3C). Taken together, these results suggest that both the intrinsic and extrinsic apoptotic pathway are activated in TG-stimulated macrophage cell death.

\section{Caspase-2 and -1 mediate activation of apoptotic caspases in} TG-stimulated THP-1 macrophage cell death

It has been reported that caspase-1, originally known for its involvement in pyroptotic cell death, induces the activation of caspase-7, which is mainly associated with apoptotic cell death (16). Furthermore, recent studies have shown that caspase-2 modulates the activation of apoptotic caspase-9 and $-8(6,17)$. Therefore, we investigated whether caspase- 2 and -1 are 
A

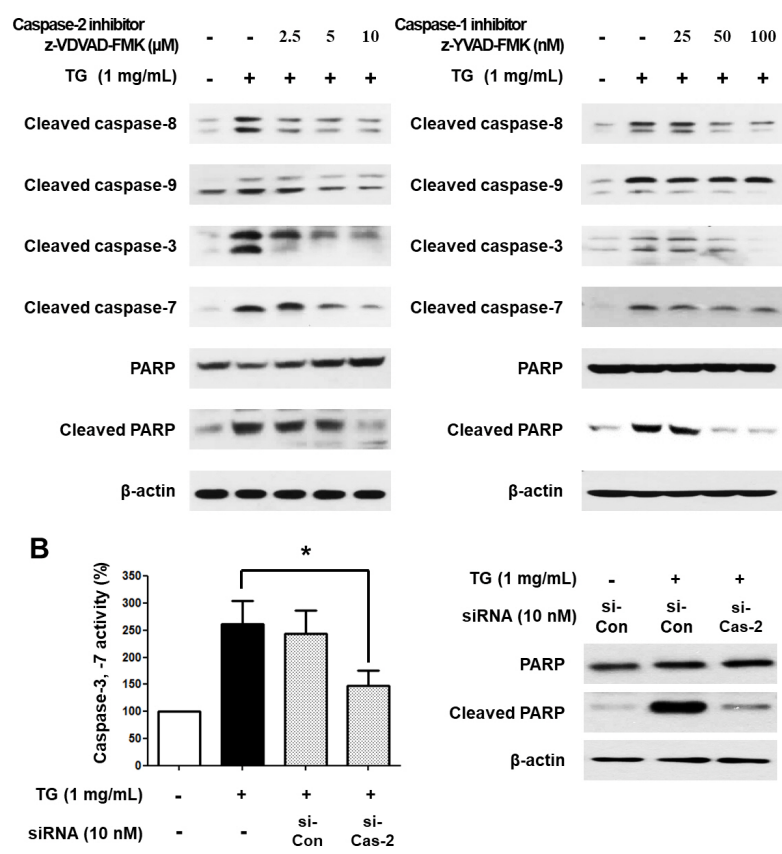

Fig. 4. Activation of apoptotic caspases is mediated via caspase- 1 and caspase-2 in TG-treated THP-1 macrophages. (A) THP-1 macrophages were treated with TG in the presence of the caspase-2 inhibitor z-VDVAD-FMK for 24 h. Cleavage of caspase-8, -9, -3, -7, and PARP was detected by Western blotting. THP-1 macrophages were transfected with control siRNA or caspase-2 siRNA for $24 \mathrm{~h}$ and incubated with or without TG for an additional $24 \mathrm{~h}$. (B) THP-1 macrophages were transiently transfected with the control siRNA or caspase-2 siRNA for $24 \mathrm{~h}$ and incubated with TG for an additional $24 \mathrm{~h}$. Caspase-3/7 activity was assessed (left) and cleavage of PARP was detected by Western blotting (right). (C) THP-1 macrophages were treated with TG in the presence of the caspase-1-specific inhibitor z-YVAD-FMK for $24 \mathrm{~h}$. Cleavage of caspase-8, -9, -3, -7, and PARP was detected by Western blotting. Data are expressed as the mean \pm SEM of three independent experiments. The $P$ values were determined with Student's t-test. ${ }^{*} \mathrm{P}<0.05$.

involved in the activation of caspase-3, -7, -8, and -9 in TG-induced macrophage cell death. Specifically, THP-1 macrophages were treated with TG in the absence or presence of caspase- 1 or -2 specific inhibitors for $24 \mathrm{~h}$. As shown in Fig. $4 \mathrm{~A}$, treatment with a caspase-2 specific inhibitor decreased cleavage of caspase-8/9, -3/7, and PARP in a dose-dependent manner. In addition, when caspase-2 was knocked down in THP-1 macrophages using siRNA, caspase-3/7 activity and cleaved PARP levels were decreased (Fig. 4B). Inhibition of caspase-1 also reduced cleavage of caspase-8/9, -3/7, and PARP (Fig. 4C). Taken together, these results suggest that caspase-2 and caspase- 1 are upstream of the apoptotic caspases, thereby implicating them in the activation of apoptotic caspases in TG-stimulated THP-1 macrophage cell death.

\section{DISCUSSION}

Plaque formation is an important step in the development of atherosclerosis. One of the key events in plaque formation and instability is macrophage cell death. It has been demonstrated that uptake of TG into macrophages contributes to the formation of a necrotic core that is conducive to the inflammatory process, as well as plaque development and vessel rupture (3, $20,21)$. However, the mechanisms by which TG induces macrophage cell death has yet to be fully revealed. We previously reported that caspase- 1 is involved in TG-induced macrophage cell death (14). Herein, we investigated the role of caspase-2 in TG-stimulated cell death of macrophages. We found that i) caspase-2 is involved in TG-induced macrophage cell death, ii) TG activates both intrinsic and extrinsic apoptotic caspases, and iii) caspase- 2 is an upstream molecule of caspase- 1 that induces activation of apoptotic caspases in TG-stimulated macrophage cell death.

Caspase-1 is the central regulator of inflammation and pyroptotic cell death $(10,15)$. Caspase-1 activation has been detected in inflammatory vascular lesions in patients suffering from acute coronary events $(5,22)$. Previously, we showed that caspase-1 is associated with TG-induced macrophage cell death, an important event in chronic vascular diseases (14). Previous studies also suggest that pyroptotic cell death may be associated with chronic vascular disease. In the present study, we found that caspase- 1 activates apoptotic caspases that are associated with apoptotic cell death in TG-induced macrophage cell death. Recently, caspase- 1 was found to be important for apoptotic cell death in response to several stimuli $(15,16,23$, 24). Several studies demonstrated that caspase-1 deficiency reduces processing of caspase- 9 and -3 , cleavage of Bid, and release of cytochrome $c$ in mouse models $(15,23)$. Another report showed that caspase-7 is a substrate of caspase-1 (16, 24). Taken together, these results suggest that TG-induced macrophage cell death is processed both through pyroptotic and apoptotic pathways, and that caspase- 1 plays a role in both pyroptotic and apoptotic macrophage cell death in response to TGs.

Caspase-2 is associated with cell death induced by a variety of stressors such as DNA damage, ROS, and secreted TNF- $\alpha$ (17). A recent report showed that activation of caspase- 1 is driven by the activation of caspase-2 in Brucella abortus RB51-induced hybrid cell death (6). Other studies have shown that caspase-2 induces the truncation of Bid (tBid), which plays a role in the release of cytochrome $c$, resulting in activation of caspase- 9 and caspases-3/7 (6, 9). In addition, studies have shown that the activation of caspase-8 is dependent on caspase-2 $(6,17,25)$. Meanwhile, other reports indicate that caspase-2 is able to cleave PARP directly, resulting in disintegration of DNA-repair processing and initiation of apoptosis $(18,26)$. Based on these studies, we examined whether caspase-2 is also involved in TG-induced macrophage cell death. Our results showed that caspase- 2 is associated 
with TG-stimulated cell death and induces activation of caspase-1, which in turn activates apoptotic caspases.

Caspase- 9 and -8 have been linked to intrinsic (mitochondrial) and extrinsic apoptotic pathways, respectively $(5,6)$. In this study, we found that cleavage of caspase-9 was almost completely recovered by inhibiting caspase-2 in TG-treated THP-1 macrophages. However, inhibition of caspase-1 did not completely prevent cleavage of caspase-9 (Fig. 4). On the other hand, cleavage of caspase- 8 was recovered in response to treatment with both caspase- 1 and -2 inhibitors (Fig. 4). Taken together, these results suggest that caspase-2 is involved in both the intrinsic and extrinsic apoptotic pathway, while, caspase- 1 is primarily involved in the extrinsic apoptotic pathway in TG-treated THP-1 macrophage cell death.

In conclusion, we report the novel finding that caspase-2 is implicated in TG-induced macrophage cell death. Based on our results, we suggest that both the pyroptotic and apoptotic pathways are associated with TG-stimulated macrophage cell death. Although further studies are needed to investigate other possible pathways of TG-induced macrophage cell death, we expect that the results of this study will contribute to a better understanding of the role of caspase- 2 in cell death and provide a basis for future studies on the role of caspase- 2 in the development of chronic vascular diseases such as atherosclerosis.

\section{MATERIALS AND METHODS}

\section{Materials}

TG emulsion (Lipofundin $\left.{ }^{(}\right)$MCT/LCT 20\%) was purchased from B. Braun Melsungen AG (Melsungen, Germany). Lipofundin ${ }^{\mathbb{R}}$ MCT/LCT $20 \%$ was used to deliver TG into cells as described previously (27). Hereafter, Lipofundin ${ }^{\circledR}$ MCT/LCT $20 \%$ is referred to as TG for convenience. The caspase-1 substrate Ac-YVAD-p-nitroanilide (Ac-YVAD-pNA) was purchased from Biomal (Plymouth Meeting, PA, USA). The caspase-2 substrate Ac-VAVAD-pNA was purchased from Sigma-Aldrich (St. Louis, MO, USA). The caspase-3/7 substrate Ac-DEVD-pNA was purchased from Enzo Life Sciences (Farmingdale, NY, USA). The caspase-1 specific inhibitor Z-YVAD-FMK and caspase-2 specific inhibitor z-VDVAD-FMK were purchased from BioVision (Mountain View, CA, USA). Antibodies specific for caspases-1, - -3, -7, -8, and -9, as well as PARP, were purchased from Cell Signaling Technology (Danvers, MA, USA).

\section{Cell culture}

The THP-1 human acute monocytic leukemia cell line (ATCC, Manassas, VA, USA) was grown in RPMI 1640 media supplemented with $10 \%$ fetal bovine serum (FBS) and penicillinstreptomycin and maintained at $37^{\circ} \mathrm{C}$ in a humidified atmosphere with $5 \% \mathrm{CO}_{2}$. To induce differentiation of THP-1 cells into macrophages, cells were seeded in 6-well plates at a density of $1 \times 10^{6}$ cells/well and treated with $200 \mathrm{nM}$ PMA for $48 \mathrm{~h}$.

\section{siRNA transfection}

Caspase-2 siRNAs (5'-UGGAAGUAUUUGAGAGAGAdTdT-3') were synthesized by ST PHARM (Seoul, Korea). Transfections were performed with Lipofectmine 2000 (Invitrogen, Carlsbad, CA, USA) according to the manufacturer's protocol.

\section{Trypan blue dye exclusion assay}

To enumerate viable cells, cells were trypsinized and $10 \mu \mathrm{l}$ of $0.4 \%$ trypan blue stain solution was mixed with $10 \mu \mathrm{l}$ of the trypsinized cells at a 1:1 ratio. Non-stained cells in the resulting mixture were counted using a hemocytometer.

\section{Measurement of caspase activity}

The activity of caspase- 1 , caspase- 2 , and caspase- $3 / 7$ was determined as previously described $(28,29)$. Briefly, cells were lysed with PBS buffer containing $1 \%$ Triton X-100 and then centrifuged at $19,000 \mathrm{~g}$ for $10 \mathrm{~min}$ at $4^{\circ} \mathrm{C}$. The supernatant was collected and the total protein concentration was quantified. To detect caspase- 1 activity, $90 \mu \mathrm{g}$ of protein sample was combined with $200 \mu \mathrm{M}$ of the caspase-1 substrate Ac-YVAD-pNA in $150 \mu$ of PBS. To detect caspase-2 activity, protein was combined with the caspase-2 substrate AcVAVAD-pNA in PBS. To detect caspase-3/7 activity, protein was combined with the caspase-3/7 substrate Ac-DEVD-pNA in PBS. Reactions were incubated for $3 \mathrm{~h}$ at $37^{\circ} \mathrm{C}$, and the activity was determined by measuring the absorbance at 405 nm.

\section{Western-blot analysis}

Cells were washed with PBS and lysed at $4^{\circ} \mathrm{C}$ with lysis buffer containing 1\% Triton X-100, protease inhibitor cocktail (SigmaAldrich), phosphatase inhibitor cocktail (Roche, Mannheim, Germany), and PBS. Lysates were clarified and the supernatants were subjected to Western blotting as described previously (30).

\section{Statistical analysis}

Statistical analysis was performed using GraphPad Prism 5 (GraphPad Software Inc., San Diego, CA, USA). The P values were calculated using Student's t-test. Values are shown as the mean and standard error of the mean (SEM). Each experiment was conducted three times and the data were pooled for analysis. Differences were considered to be statistically significant at $* \mathrm{P}<0.05,{ }^{*} * \mathrm{P}<0.01$, or $* * * \mathrm{P}<0.001$.

\section{ACKNOWLEDGEMENTS}

This work was supported by the 2015 Gimcheon University Research Grant.

\section{CONFLICTS OF INTEREST}

The authors have no conflicting interests. 


\section{REFERENCES}

1. Kastelein JJ, van der Steeg WA, Holme I et al (2008) Lipids, apolipoproteins, and their ratios in relation to cardiovascular events with statin treatment. Circulation 117, 3002-3009

2. Sofer O, Fainaru M, Schafer Z and Goldman R (1992) Regulation of lipoprotein lipase secretion in murine macrophages during foam cell formation in vitro. Effect of triglyceride-rich lipoproteins. Arterioscler Thromb 12, 1458-1466

3. Aronis A, Aharoni-Simon M, Madar Z and Tirosh O (2009) Triacylglycerol-induced impairment in mitochondrial biogenesis and function in J774.2 and mouse peritoneal macrophage foam cells. Arch Biochem Biophys 492, 74-81

4. Gotto AM, Jr. (1998) Triglyceride: the forgotten risk factor. Circulation 97, 1027-1028

5. Elmore S (2007) Apoptosis: a review of programmed cell death. Toxicol Pathol 35, 495-516

6. Bronner DN, O'Riordan MX and He Y (2013) Caspase-2 mediates a Brucella abortus RB51-induced hybrid cell death having features of apoptosis and pyroptosis. Front Cell Infect Microbiol 3, 83

7. Albert ML (2004) Death-defying immunity: do apoptotic cells influence antigen processing and presentation? Nat Rev Immunol 4, 223-231

8. Brennan MA and Cookson BT (2000) Salmonella induces macrophage death by caspase-1-dependent necrosis. Mol Microbiol 38, 31-40

9. Jesenberger V, Procyk KJ, Yuan J, Reipert S and Baccarini M (2000) Salmonella-induced caspase-2 activation in macrophages: a novel mechanism in pathogen-mediated apoptosis. J Exp Med 192, 1035-1046

10. Thornberry NA, Rano TA, Peterson EP et al (1997) A combinatorial approach defines specificities of members of the caspase family and granzyme B. Functional relationships established for key mediators of apoptosis. J Biol Chem 272, 17907-17911

11. Cohen GM (1997) Caspases: the executioners of apoptosis. Biochem J 326 (Pt 1), 1-16

12. Tewari M, Quan LT, O'Rourke K et al (1995) Yama/CPP32 beta, a mammalian homolog of CED-3, is a CrmAinhibitable protease that cleaves the death substrate poly(ADP-ribose) polymerase. Cell 81, 801-809

13. Aflaki E, Radovic B, Chandak PG et al (2011) Triacylglycerol accumulation activates the mitochondrial apoptosis pathway in macrophages. J Biol Chem 286, 7418-7428

14. Son SJ, Rhee KJ, Lim J, Kim TU, Kim TJ and Kim YS (2013) Triglyceride-induced macrophage cell death is triggered by caspase-1. Biol Pharm Bull 36, 108-113

15. Zhang $W H$, Wang $X$, Narayanan $M$ et al (2003) Fundamental role of the Rip2/caspase-1 pathway in hypoxia and ischemia-induced neuronal cell death. Proc Natl Acad Sci U S A 100, 16012-16017

16. Lamkanfi M, Kanneganti TD, Van Damme P et al (2008) Targeted peptidecentric proteomics reveals caspase-7 as a substrate of the caspase- 1 inflammasomes. Mol Cell Proteomics 7, 2350-2363

17. Puccini J, Dorstyn L and Kumar S (2013) Caspase-2 as a tumour suppressor. Cell Death Differ 20, 1133-1139

18. Fava LL, Bock FJ, Geley S and Villunger A (2012) Caspase-2 at a glance. J Cell Sci 125, 5911-5915

19. Bouchier-Hayes L and Green DR (2012) Caspase-2: the orphan caspase. Cell Death Differ 19, 51-57

20. Aronis A, Madar Z and Tirosh O (2008) Lipotoxic effects of triacylglycerols in $\mathbf{7 7 4 4 . 2}$ macrophages. Nutrition 24, 167-176

21. Lim J, Kim YS, Kim SH et al (2013) Triglyceride enhances susceptibility to TNF- $\alpha$-induced cell death in THP-1 cells. Genes \& Genomics 36, 87-93

22. Kolodgie FD, Narula J, Burke AP et al (2000) Localization of apoptotic macrophages at the site of plaque rupture in sudden coronary death. Am J Pathol 157, 1259-1268

23. Plesnila N, Zinkel S, Le DA et al (2001) BID mediates neuronal cell death after oxygen/ glucose deprivation and focal cerebral ischemia. Proc Natl Acad Sci U S A 98, 15318-15323

24. Yeretssian G, Labbe K and Saleh M (2008) Molecular regulation of inflammation and cell death. Cytokine 43, 380-390

25. Huang YT, Huang YH, Hour TC, Pan BS, Liu YC and Pan $\mathrm{MH}$ (2006) Apoptosis-inducing active components from Corbicula fluminea through activation of caspase-2 and production of reactive oxygen species in human leukemia HL-60 cells. Food Chem Toxicol 44, 1261-1272

26. Stefanis L, Troy CM, Qi H, Shelanski ML and Greene LA (1998) Caspase-2 (Nedd-2) processing and death of trophic factor-deprived $\mathrm{PC} 12$ cells and sympathetic neurons occur independently of caspase-3 (CPP32)-like activity. J Neurosci 18, 9204-9215

27. Lim J, Kim SH, Kang YW et al (2014) Triglyceride up-regulates expression of ABCG1 in PMA-induced THP-1 macrophages through activation of JNK and p38 MAPK pathways. Biomed Sci Lett 20, 237-243

28. Joo D, Woo JS, Cho KH et al (2016) Biphasic activation of extracellular signal-regulated kinase (ERK) $1 / 2$ in epidermal growth factor (EGF)-stimulated SW480 colorectal cancer cells. BMB Rep 49, 220-225

29. Imre G, Heering J, Takeda AN et al (2012) Caspase-2 is an initiator caspase responsible for pore-forming toxinmediated apoptosis. EMBO J 31, 2615-2628

30. Jo HS, Kim DS, Ahn EH et al (2016) Protective effects of Tat-NQO1 against oxidative stress-induced HT-22 cell damage, and ischemic injury in animals. BMB Rep 49, 617-622 